\title{
Maintaining contact with blue-green spaces during the COVID-19 pandemic associated with positive mental health
}

\author{
Sarai Pouso*a; Ángel Borja ${ }^{a}$; Lora E. Fleming ${ }^{b}$; Erik Gómez-Baggethun ${ }^{c, d}$; Mathew P. \\ White $^{\mathrm{b}}$; María C. Uyarra ${ }^{\mathrm{a}}$
}

a AZTI, Marine Research, Basque Research and Technology Alliance (BRTA). Herrera Kaia, Portualdea z/g, 20110 Pasaia - Gipuzkoa, Spain

b European Centre for Environment and Human Health, University of Exeter Medical School, Exeter, UK.

c Department of International Environment and Development Studies (Noragric), Norwegian University of Life Sciences (NMBU), P.O. Box 5003, Ås N-1432, Norway

d Norwegian Institute for Nature Research (NINA), Gaustadalleen 21, Oslo 0349, Norway

${ }^{*}$ Corresponding author: Sarai Pouso (spouso@azti.es)

\begin{abstract}
There is growing evidence that exposure to the natural world (blue-green spaces) has potential benefits for mental health and well-being. The COVID-19 pandemic and the measures adopted to control it provide a natural experiment to investigate the links between nature exposure and mental health under extreme conditions. Using a survey distributed online and based on 6,080 responses, we tested three hypotheses: (1) people will show different levels of symptoms of depression and anxiety depending on the level of lockdown (severity) and ability to maintain contact with outdoor spaces; and (2) where access to outdoor public spaces was restricted, those with access to private outdoor spaces (2a) or even a green-blue nature view (2b) will show fewer symptoms of depression and anxiety and a more positive mood. Lockdown severity significantly affected mental health, while contact with nature helped people to cope with these impacts. The buffering effect of nature was especially relevant for those under strict lockdowns. People perceived that nature helped them to cope with lockdown measures; and emotions were more positive among individuals with accessible outdoor spaces and blue-green elements in their views. These findings can help decision-makers in developing potential future lockdown measures to mitigate the negative impacts, helping people to be more resilient and maintain better mental health.
\end{abstract}

\section{Keywords}

Ecosystem services, nature's contributions to people, anxiety, depression, green-blue infrastructure 


\section{Introduction}

In December 2019, a new coronavirus (SARS-CoV-2) and associated disease (COVID-19) were identified in China, and quickly spread to the rest of the world (1). This led to a rapid increase of infections and deaths, which jeopardized the capacity of public health systems all over the world ${ }^{1}$ and governments took measures to contain the disease.

These measures have been highly variable among countries but the most common were border and school closures, teleworking, and, particularly, measures restricting the movement of people, including so called "lockdowns" $(2,3)$. In Europe, the first country to enact a national lockdown was Italy ( $11^{\text {th }}$ March), followed by Spain ( $15^{\text {th }}$ March), France $\left(17^{\text {th }}\right.$ March), Germany (22 ${ }^{\text {nd }}$ March), and the United Kingdom (23 $3^{\text {rd }}$ March). The stringency of the implemented measures varied among countries. The three major approaches were: Level 1- people were not allowed to leave their homes except for activities such as essential jobs, buying food and medicines, emergencies or walking the dog (e.g. China, Italy or Spain); Level 2- severe lockdown but with certain time for outdoor exercise (e.g. France, United Kingdom); and Level 3- the limitation of movements as recommendations rather than binding rules (e.g. Scandinavian countries).

The implementation of physical distancing and lockdowns are likely responsible for having saved millions of lives; with estimates of $>3$ million lives saved in 11 European countries alone (4). However, these interventions are likely to have led to many unintended consequences and the COVID-19 pandemic has provided a natural experiment. Thus, parallel to the fast-moving medical research to find effective treatments and a vaccine, studies on how the pandemic and the subsequent lockdown measures are impacting the environment $(5,6)$, the economy $(7)$ and people's mental health (8-10) have also been conducted.

Central to COVID-19 restrictions in most countries has been physical distancing and even "self-isolation" or "quarantine" from others. There is very strong evidence suggesting that isolation from others can damage mental health (11-13). Thus, it is not surprising that symptoms of depression and anxiety (already some of the most common mental health disorders) (14), have increased in the early stages of lockdown $(15,16)$. However, there is also emerging evidence that individual's psychological resilience can help to cope and adapt to adverse circumstances $(17,18)$. Also, that contact with nature can buffer or mitigate against the negative effects of social isolation on mental health $(19,20)$, an effect that may have been especially important during lockdown (21).

Indeed, there is a growing literature showing that exposure to blue-green spaces (e.g. urban parks, woodlands, rivers and the coast), has a range of potential benefits for mental health and well-being (22-26), also referred to as cultural ecosystems services (27). Exposure can come in three ways: direct contact, e.g. deliberately visiting a park for recreation; indirect contact, e.g. window views of natural spaces; and incidental contact e.g. passing through a park when commuting to work (28). To date the strongest evidence in support of mental health benefits has been for direct contact in natural settings, with benefits to general $(29,30)$ and clinical $(31,32)$ populations. A large UK study with over 20,000 people estimated two hours/week outdoors in bluegreen spaces as a critical threshold, below which well-being decreases significantly (33). But indirect contact, by having a window view of nature, and especially blue spaces, has also been found to be positively associated with better mental health, even once socio-economic factors have been taken into account (34-36). Importantly for the COVID-19 crisis, research also suggests that maintaining contact with nature during stressful life events, such as relationship breakdown or job loss, can "buffer" individuals against stress; with those living in greener areas reporting fewer mental and physical symptoms of poor-health during, and shortly after, the stressful event (37).

${ }^{1}$ https://www.ecdc.europa.eu/sites/default/files/documents/COVID-19-guidance-health-systems-contingencyplanning.pdf 
Under the probable scenario of a second wave of COVID-19 $(38,39)$ and/or spread of new emerging diseases in the near future, we need to understand the specific factors that positively contributed to mental health during lockdown, so measures/strategies can be better designed in future.

The aim of the current study was to test whether, during the first wave of the COVID-19 outbreak, people who maintained direct and/or indirect contact with outdoor spaces coped better with lockdown measures in terms of fewer symptoms of poor mental health (i.e. depression and anxiety) and better maintenance of positive mood. To achieve this aim, an online self-report survey was distributed between April-May 2020, to people around the world and under different lockdown scenarios.

We tested the following three hypotheses: 1) People will show different levels of symptoms of depression and anxiety depending on the level of lockdown and whether they were able to maintain contact with public outdoor nature spaces; 2) Where access to public outdoor nature spaces was restricted, those with access to outdoor spaces such as a garden or balcony (2a) or even a green/blue nature view (2b) will show fewer symptoms of depression and anxiety, and a more positive mood.

\section{Results}

A total of 6,895 responses were received from the online survey, with a usable sample of 6,080 responses, after the application of filters (Supplementary Information (SI) Figure S1). We obtained responses from 77 countries, with the highest representation corresponding to the European countries of Spain ( $n=3,704$ participants; $61 \%)$, United Kingdom $(n=451 ; 7.4 \%)$ and Germany ( $n$ $=324 ; 5.3 \%)$.

All 6,080 participants were used to test the first Hypothesis. The subsample of respondents from Spain in severe lockdown (Level 1) ( $n=3,403 ; 56.0 \%$ ) was used to test Hypothesis 2. This Spanish subsample was selected as in countries with less strict lockdown measures (levels 2 and 3 ), the effects on mental health of residence characteristics, and more precisely, the effect of accessibility to outdoor spaces and nature views from home, could be biased by a more freely available access to nature and public outdoor spaces for recreational reasons. Furthermore, the high representation of Spain in the total sample allowed for a more in-depth analysis. The socioeconomic characteristics of the sample and subsample are summarized in SI Table S1.

Lockdown severity positively associated with poor mental health. Mental health was assessed using the 4-item Patient Health Questionnaire (PHQ-4) screening scale, composed by the 2-item PHQ-2 for depression and the 2-item Generalized Anxiety Disorder scale (GAD-2) for anxiety. Supporting our first hypothesis, people who were in Level 1 countries reported more symptoms of depression (Chi-squared $X^{2}(2)=27.501 ; p<0.001$ ) and anxiety (Chi-squared $X^{2}(2)=10.234$; $\mathrm{p}=0.006$ ), and greater signs of poor mental health overall (Kruskal Wallis $\mathrm{H}(2)=56.025 ; \mathrm{p}<0.001$; (Table 1). Specifically, the proportion of people who reported symptoms considered indicative of being at risk of depression, i.e. scores of $\geq 3$ on the PHQ-2 scale, were $25.4 \%$ for Level 1 vs. $19.1 \%$ and $20.4 \%$ in Levels 2 and 3, respectively. The pattern for anxiety (GAD-2 scores $\geq 3$ ) was similar: Level 1, 28.8\%; Level 2, 26.4\%; and Level 3, 23.6\%). In terms of the overall PHQ-4 scale $23.7 \%$ of respondents in Level 1 reported 'Moderate' or 'Severe' symptoms of poor mental health vs. $17.6 \%$ in Level 2 and 18.3\% in Level 3. For the PHQ-2 and PHQ-4 scales, post-hoc tests confirmed that people in Level 1 were significantly more likely to have symptoms of mental health issues than people in less strict lockdowns (Levels 2 and 3) (Table 1). Regarding GAD-2 for anxiety screening, 
there was a higher percentage of individuals at risk in Level 1 than in Levels 2-3, but the differences were only significant between Level 1 and 3 (Table 1).

To explore if, in addition to lockdown levels, other factors may have determined the prevalence of depression and anxiety, logistic Generalized Linear Models (GLMs) were developed, using the PHQ-2 and GAD-2 scale results as dependent variables. The models were built with three independent variables: level of lockdown (Levels 1,2,3), access to outdoor spaces (yes, no), and natural elements in views from home (yes, no). The unadjusted models (Table 2) supported hypothesis 1, less strict lockdown levels, greater access to private outdoor spaces, and nature views from home, were all associated with lower symptoms of depression and anxiety. The inclusion of socioeconomic variables (adjusted models, Table 2), did not alter the general pattern.

Several residence characteristics were predictors of symptoms of both depression and anxiety (Table 2). Having greater home space (i.e. rooms per individuals under lockdown), accessible private outdoor areas, and nature views were all associated with significantly lower odds of depression and anxiety symptoms. The more psychologically-resilient the individual (measured with the Brief Resilience Coping Scale BRCS), the lower the odds for depression (OR $=0.83 ; 95 \%$ $\mathrm{Cl} 0.81-0.85 ; \mathrm{p}<0.001)$ and anxiety $(\mathrm{OR}=0.88 ; 95 \% \mathrm{Cl} 0.86-0.90 ; \mathrm{p}<0.001)$. Regarding socioeconomic characteristics, women, younger adults, and pet owners were more likely to show symptoms of depression and anxiety than men, older people and people who did not own a pet. Other variables specifically affected either depression or anxiety symptoms; e.g. people who changed their employment status during COVID-19 (regardless of the type of change) were more likely to show symptoms of depression than respondents whose employment status did not change, but the effect was not significant for anxiety. On the other hand, spending the lockdown with people with special care needs was linked to higher odds for anxiety, while the effect for depression was not significant.

In the GLMs built with people in Level 1 (adjusted models, Table 2), the effect of accessibility to outdoor spaces and nature views was significant and strongly positive for reducing the likelihood of exhibiting symptoms of depression (access to outdoors OR=0.67; $95 \% \mathrm{Cl} 0.57$ $0.80 ; p<0.001$; and nature views $\mathrm{OR}=0.77 ; 95 \% \mathrm{Cl} 0.65-0.92 ; \mathrm{p}<0.01$ ) and anxiety (access to outdoors $\mathrm{OR}=0.77 ; 95 \% \mathrm{Cl} 0.66-0.90 ; p<0.01$; nature views $\mathrm{OR}=0.78 ; 95 \% \mathrm{Cl} 0.67-0.91 ; \mathrm{p}<0.01)$. However, for GLMS built with individuals in Levels 2-3, only nature views had a positive effect in depression $(\mathrm{OR}=0.77 ; 95 \% \mathrm{Cl} 0.61-0.97 ; \mathrm{p}<0.05)$.

Nature contact 'buffers' effect of lockdown on mental health. Supporting Hypothesis 2, when lockdown measures included strict restrictions to access outdoor public spaces (i.e. people in Spain under lockdown Level 1), individuals who had direct access to outdoor spaces from their residence (e.g. balcony, garden) and individuals with mixed or natural views from the window (e.g. green/blue spaces) showed lower symptoms of depression and anxiety than individuals with no access to outdoor spaces and with limited or urban views (Table 3). The type of accessible outdoor space and type of view were related to the likelihood of exhibiting symptoms of mental disorders (for PHQ4 values vs. type of outdoor space or vs. type of view, Kruskal Wallis Tests $p<0.001$; for PHQ-2 and GAD-2 values vs. type of outdoor spaces or vs. type of view, all chi-squared tests $p<0.001$ ). Post hoc tests confirmed that people with no accessible outdoor spaces had higher odds of clinically important symptoms of depression (PHQ-2 post hoc test $\mathrm{p}<0.05)$ and overall mental health (PHQ-4 post hoc Dunn's Test $\mathrm{p}<0.05$ ) than people with any of the three kinds of accessible outdoor spaces (balcony, other private spaces [e.g. private garden], shared or public spaces). Individuals with no nature elements in their views (i.e. limited or urban views) had higher odds of clinically important symptoms of depression (PHQ-2 post hoc tests $\mathrm{p}<0.05)$ and higher PHQ-4 scores (Dunn's Test $p<0.05$ ) than individuals with mixed or natural views. Regarding anxiety (GAD-2), results suggest that people were more likely to be $\geq 3$ threshold when they did not have accessible outdoor spaces and when they had limited or urban views. However, for GAD-2 values, the post hoc test results were not conclusive, neither for outdoor spaces (none vs. shared or public spaces, 
$\mathrm{p}<0.05$ ) nor for views (limited or urban views vs. natural views, $\mathrm{p}<0.05$ ). Regression models confirmed the overall importance of the type of outdoor spaces and the type of views for the likelihood of showing symptoms of depression and anxiety (SI Table S2). However, in the adjusted models for depression, the effect of nature views shifted to $p<0.05$, indicating that no clear positive effect was found in this type of view over the reference level "limited or urban views".

People perceived that nature helped them to cope better with lockdown measures. In connection with hypothesis 2, respondents in strict lockdown not only showed lower odds of symptoms of depression and anxiety depending on the type of accessible outdoor space and view, but also perceived the positive effect that those spaces and views had in their ability to cope with lockdown restrictions. The self-reported contribution of accessible outdoor spaces (Table 4) was more positive for people with access to a private space (e.g. garden, swimming pool) $(\mathrm{M}=4.50 \pm 0.94)$ than for people with access to a balcony $(\mathrm{M}=4.15 \pm 1.02)$ and people with access to shared or public outdoor spaces $(M=4.27 \pm 1.14)$. The differences were significant after the ANOVA test $(F(2,2281)=25.96 ; p<0.001)$ and Tukey's post hoc test $(p<0.01$ for private space vs. balcony and private space $v s$. shared or public space). Regarding views, the self-reported contribution was significantly different depending on the view type (ANOVA test $F(2,3400)=533.5 ; p<0.001$ ), with a significant decreasing effect from natural views $>$ mixed views $>$ limited/urban views (Tukey's post hoc, $p<0.001$ for the three comparisons) (Table 4). Adjustment for socioeconomic variables did not change the main results, confirming that the self-reported contribution was dependent on the type of view and on the type of accessible outdoor space (SI Table S3).

Emotions were more positive among individuals with accessible outdoor spaces and natural elements in views. Considering that the PHQ-4 scale focuses on depression and anxiety, and that the lockdown situation may have caused a greater diversity of impacts, respondents were also asked to self-assess their emotions before and during lockdown. During lockdown, the most frequently mentioned word was "bored" $(\mathrm{n}=559)$, while "optimistic" was the most frequently mentioned to characterise the remembered emotional situation pre-lockdown $(n=1,177)$ (SI Table S4). After the classification of emotions in a seven core type-emotions (i.e. angry, bad, disgusted, fearful, happy, sad, surprised), results suggest that the emotional situation of people in Spain under lockdown Level 1 worsened after the adoption of lockdown measures (during lockdown vs. before lockdown, chi-square $p<0.001$ ), with a decrease from $71 \%$ to $26.2 \%$ in the emotions classified inside the "happy" core-emotion (Figure 1). During lockdown, individuals with natural elements in their views mentioned more positive emotions ("happy" accounts for $30.1 \%$ ) than individuals with limited or urban views (23.3\%). No significant differences were encountered between view types in the emotions reported to describe the emotional situation pre-lockdown (chi-squared $p>0.05$ ), confirming that having natural elements in the view may have acted as a buffer against the negative consequences of lockdown. Regarding outdoor spaces, individuals with accessible outdoor spaces reported more positive emotions than individuals with no accessible outdoor spaces; however, the difference was significant both during and before lockdown, and therefore, differences in emotions cannot be directly linked to the lockdown situation.

\section{Discussion}

There are relatively few studies demonstrating that ecosystem services assessments can be expanded to include their potential benefits on human mental health and well-being (23). However, there is a growing body of evidence revealing the value of nature experiences for mental health $(19,22,24,35,40)$. With rapid urbanization and declines in human contact with nature, as well as the potential increase of pandemics with global change, understanding how ecosystem services can provide benefits in terms of mental health and human well-being during new lockdowns will assist public health and other decision makers to take informed decisions on the type of measures to adopt. Supporting our three hypotheses, greater lockdown severity was associated with a greater likelihood of exhibiting symptoms of mental health disorders. However, these symptoms were lower in the most severe lockdown scenario when individuals still had access to private outdoors spaces 
such as balconies or gardens, and when their views from home included natural elements. Furthermore, people self-identified the positive contributions of nature views and outdoor spaces and maintained a more positive attitude in terms of mood when they had access to these resources during lockdown.

Previous studies have explored the physical and mental benefits of spending time outdoors (33), and the buffering effect of nature in individuals suffering from social isolation (19) or stressful life events (37). In line with those studies and confirming our first hypothesis, our results showed the clear negative effect of severe confinement on mental health, with people who had restricted access to outdoor public spaces (Level 1) more likely to show symptoms of mental health disorders than people who had partial (Level 2) or no restriction (Level 3) to access to outdoor spaces. Furthermore, the lack of significant differences in terms of symptoms of depression and anxiety between people in Level 2 and in Level 3 highlights the important role of contact with nature for maintaining good mental health: even when authorities fixed a limited time to be outdoors (level 2), this allowance had an important effect in reducing the likelihood of mental health issues.

During COVID-19, among people who could spend free time outdoors, the importance of contact with nature from home to reduce the likelihood of suffering from depression and anxiety was less important. Indeed, the buffering effect of nature (access to outdoor private spaces and the views from windows) in the regression models was only clear for people at Level 1 of lockdown. This result can be related to the variables selected to explore the contact with nature in the regressions: access to outdoor spaces from home (yes/no) for direct contact, and the presence of natural elements in views (yes/no) for indirect contact. These variables, related to residence characteristics, are likely less important for individuals at Level 2 and 3 , as they were able to maintain access to outdoor spaces, e.g. public parks. Furthermore, the measures adopted in countries in Level 2 and 3 (e.g. switching to home office working or being placed on furlough) might have led to an increase in the time available and the possibility to spend it in natural outdoors setting, with positive effect on well-being (21). For example, a recent study suggested that time spent outdoors increased in Norway during COVID-19 lockdown (41).

Maintaining contact with nature has been reported to be beneficial in times of stress (37), and exploring this effect in people who spent the first wave of COVID-19 under strict lockdown (e.g. Spain) is especially relevant. The measures adopted in Spain to flatten the curve of the disease were very restrictive (3), with inhabitants spending nearly two months (March-May 2020) under a stay-at-home order. In this context, our findings confirmed that having accessible outdoor spaces and natural elements in views from home were key factors associated with a reduced likelihood of exhibiting symptoms of depression and anxiety, with differences in the effect across types of accessible outdoor spaces and views. People who had access to a private outdoor space (e.g. garden, swimming pool) coped better with lockdown limitations in terms of mental health, compared to individuals who had balconies or shared/public spaces. The higher positive effect of private outdoor spaces compared to balconies could be related to a higher space availability and a higher naturalness of the space. The apparently more negative effect of public and shared outdoor spaces compared to private outdoor spaces could be related to the presence of people in these spaces, which can complicate the maintenance of the recommended social distance.

For types of views, mixed views (i.e. natural views plus urban views) were found to be the ones with the most positive association. Longstanding research has shown that, all else equal, people are willing to pay more for houses and hotel rooms with views of nature $(42,43)$, which can be interpreted in terms of benefits from cultural ecosystem services. Further research is needed to elucidate if under COVID-19 lockdown, there are significant differences between types of nature views, e.g. if views to blue spaces have a higher buffering effect than green spaces, as suggested by previous studies (34). Further research could also test whether there are significant differences between the type of contact maintained with nature during lockdown, e.g. by comparing passive 
contact (e.g. looking at nature through the window or sitting in the terrace) with active contact (e.g. practicing physical activities outdoors).

The PHQ-4 scale was used to study mental health vs. contact with nature during lockdown. However, as divergent results might be obtained for measured effect (PHQ-4) and human perceptions and emotions, these were further analyzed. Focusing on accessible outdoor spaces, we found a similarity between what mental health scales indicated and people's perceptions and emotions: individuals with accessible outdoor spaces were not only less likely to show symptoms of depression and anxiety, but also perceived a more positive contribution of those spaces and maintained more positive emotions than individuals with no accessible outdoor spaces. Interestingly, differences in emotions between people with and without accessible outdoor spaces existed before lockdown, suggesting that the positive effect of those spaces is also important under normal circumstances $(23,44)$. Regarding views, some discrepancies arose between what mental health scales indicated and what respondents perceived: no significant differences were found between people with mixed and natural views in the likelihood of depression symptoms; however, a more positive effect of views was perceived among individuals with natural views over those with mixed views. Regarding emotions, results suggested that the buffering effect of views was especially relevant only under severe isolation situations such as COVID-19 lockdown, as no significant differences between views were found in the emotions mentioned to describe their mood before lockdown.

Apart from severity of lockdown measures, accessibility to outdoor spaces and views from home, other factors might influence the prevalence of symptoms of poor mental health. In this study, we found that psychological resilience, age and gender were important factors predicting the likelihood of showing symptoms of depression and/or anxiety during COVID-19, with consistent effect across the different levels of lockdown. Psychological resilience reflects the adaptative capacity of an individual to respond to current or future challenges (45), and our results showed that individuals with higher resilience had lower odds of showing symptoms of depression and anxiety. Resilience is considered a developmental characteristic that can be enhanced (46), meaning that measures can be taken to psychologically prepare and protect individuals for future pandemics.

Regarding age, younger people had higher odds of depression and anxiety symptoms than older people. This is interesting, since this age group was less severely hit by the disease, with milder physical symptoms and fewer severe cases than older people. Also, considering that under normal circumstances, the odds to show symptoms of mental health disorders such as anxiety increase with age (47). Yet, our results for age are consistent with studies on mental health carried out during the COVID-19 $(15,48)$. Despite social media communication having potentially played an essential role in socialization, this age group may have a higher natural demand for physical socialization, which was lacking during lockdown. For gender, the prevalence of depression and anxiety pre-COVID-19 has been previously reported as higher among women than men (49), and our study suggests that this higher prevalence continues under extreme situations such as lockdown. Our study might be showing a confounding effect of age and gender, as young women likely had more childcare responsibilities during lockdown².

Some personal characteristics were relevant for certain lockdown levels and for depression and/or anxiety, i.e. individuals who suffered a change in employment after COVID-19 and pet owners. Many people have lost their jobs during the pandemic ${ }^{3}$, and under the expected economic crisis in the years to come it is not surprising to find a higher risk of mental health disorders among

${ }^{2}$ https://eige.europa.eu/publications/gender-equality-index-2019-report/informal-care-children-andchildcare-services

${ }^{3}$ https://www.brookings.edu/research/the-effects-of-covid-19-on-international-labor-markets-anupdate/ 
people who are undergoing an unstable employment situation (50). The higher odds for depression and anxiety found among pet owners and in Level 1 of lockdown was a surprising result, as these individuals could spend extra time outdoors to walk their pet. However, it could be related to the fact that they were proportionally more restricted, if compared to their standard walks (e.g. in some countries there were limitations on the times that one could go out to walk the pet), transforming the usually-pleasant activity into an obligation with health risks.

When interpreting our results, the limitations of the study, especially the ones related with sampling, should be considered. Due to the limited time to prepare and conduct the study, the survey could only be translated and distributed in English and Spanish. Also, to capture a sample from as many countries as possible and while the lockdown measures were in place, the snow-ball sampling technique was used. These decisions influenced the composition of the sample, with a bias towards responses from Spain and an over-representation of high-educated people. Still, as mentioned above, our results are consistent with other studies published on mental health in times of COVID-19.

This study provides evidence that maintaining contact with nature in extreme situations such as the COVID-19 pandemic may be important for mental health. This beneficial effect is linked to the idea of ecosystem services, which are defined as the ecological characteristics, functions and processes that contribute to human well-being $(51,52)$. The human benefits obtained from contact with nature are classified as cultural ecosystem services, i.e. the non-material outputs that promote physical and mental health and have positive effect on social relationships $(53,54)$. All in all, the "restorative effect of nature" analyzed in psychological studies and "non-material outputs from nature" analyzed from the ecosystem services perspective appear to be two bodies of knowledge studying the same concept from different disciplines (23). In extreme situations such as the COVID-19 pandemic, the importance of cultural ecosystem services to protect human health and well-being is more evident than ever. Indeed, the extreme situation that we all underwent (and continue to be responsive to) both individually and as a society, as well as the apparent role of nature to protect us, can be used to increase awareness of the tremendous challenges we are facing. The increasing pressures and degradation of the environment, which its most evident consequence is climate change, are risking the ecosystem services we obtain from nature and humans' survival. Indeed, the appearance of the COVID-19 pandemic has been linked to the degradation of the environment and the situation is expected to worsen with more frequent and more rapidly spreading disease outbreaks (55). Therefore, it is important to support transformative changes that protect nature and reduce the risk of suffering from new pandemics, but also to be ready to face them in the future. The first wave of COVID-19 has been an unwanted experiment from which lessons can be learnt if we analyse the (un)intended outcomes of the implemented measures. Critical analysis of lessons will help individuals and societies prepare for future coronavirus-like situations.

In this sense, our results can be valuable in the design of future lockdown measures and urban plans. We found that the lockdown measures adopted led to negative consequences in people's mental health, with clear differences across the levels of lockdown. Therefore, future measures should be designed to protect individuals from the disease and from the mental and physical consequences of social isolation and stay-at-home orders. We acknowledge that the first criteria to be considered when trying to control an epidemic or pandemic should be medical and epidemiological. But if future studies do not find clear differences in flattering the curve of the infections between countries in Level 1 and Level 2 lockdowns, the less strict Level 2 could be a more adequate approach to control the disease, while at the same time reducing the likelihood of suffering mental health disorders. However, if epidemiologic studies confirm that the spread of the disease is only avoidable under strict lockdown, we recommend health authorities to be ready for a higher prevalence of mental health disorders, especially focusing on vulnerable subgroups such as women, young people or people with no contact with nature. 
Finally, an important aspect to be considered is the difference in accessibility to nature across the population. We found that house characteristics, such as outdoor spaces and views played an important role on people's mental health during the first wave of COVID-19, with important differences across types of spaces and views. When designing future urban plans, and similar to the plans to make cities more resilient to climate change (56), houses and cities should be adapted to ensure that we live in healthy spaces, and that we are resilient to the consequences of future pandemics. For example, at the house level, it can be appropriate to ensure that new houses in densely populated areas include terrace and/or balconies ${ }^{4}$. At the city level, the plans to restore, protect and promote the public green-blue spaces and their ecosystem services, and advances towards equal accessibility to these spaces $(57,58)$, should be accompanied with measures to guarantee greater space that allows for physical distancing in times of pandemics.

\section{Materials and Methods}

Survey structure and distribution. The online survey comprised 54 questions divided into 12 sections (SI Survey). The objective was to collect responses from as many countries as possible to capture the highest diversity of lockdown conditions; therefore, the survey was designed in English and translated to Spanish. The survey was distributed using Google Forms, between $17^{\text {th }}$ April and $8^{\text {th }}$ May 2020, starting when most European countries had spent at least one month under lockdown (4) and finishing when some countries started to ease lockdown measures ${ }^{5,6}$.

The first question of the survey was related to the level of lockdown in which the respondent was when answering the survey. This question allowed for the classification of responses according to the three levels of lockdown mentioned in the introduction.

For analyzing mental health issues, the PHQ-4 screening scale was used (59). The PHQ4 scale is a self-administered survey, commonly used in primary care and in remote health surveys to detect people at risk of suffering depression and anxiety (59-61). It composed of four questions or two ultra-brief screening scales with two questions each: the GAD-2 for screening anxiety disorders (62); and the PHQ-2 for screening depression disorders (63). Respondents chose between four possible response options (from "not at all" (0) to "nearly every day" (3)). Scores for the GAD-2 and PHQ-2 range from 0-6, and for PHQ-4, from 0 to 12.

Using the PHQ-4 scale, we explored the negative effect of lockdown on symptoms of depression and anxiety; however, lockdown measures may have enhanced a much broader range of emotions. To capture emotional changes, respondents self-assessed their emotions pre- and during lockdown, using a figure designed based on Plutchik's wheel of emotions (64). The figure comprised a total of 41 emotions classified in seven core emotions (i.e., happy, sad, disgusted, angry, fearful, bad, surprised). Respondents had to select the emotions that best described their current general mood and their character under normal and before coronavirus outbreak (they were asked to select between one and three emotions in each question).

For the sociodemographic characterization of respondents, 14 questions were included regarding age, gender, marital status, country of residence, maximum education level achieved, employment status before and during COVID-19, annual gross income and pet ownership. To characterize the psychological resilience of respondents, the 4-item BRCS was included $(18,65)$. The BRCS captures how adaptable individuals are to cope with stress. To characterize the conditions of the lockdown, questions on the company of others during lockdown were included: whether they spent the lockdown alone, with kids and with people with special care needs. Also, questions on the characteristics of the residence were collected (size, views from residence and

\footnotetext{
${ }^{4}$ https://www.deia.eus/actualidad/sociedad/2020/06/19/pisos-euskadi-tendran-35-metros/1046587.html

${ }^{5} \mathrm{https://www.bbc.com/news/explainers-52575313}$

6 https://www.euronews.com/2020/03/19/coronavirus-which-countries-are-under-lockdown-and-who-s-next
} 
accessible outdoor spaces). Respondents indicated to what extent the views and the accessibility to outdoor spaces helped them to cope better with the lockdown situation, by answering two Likertscale questions (1-not at all, 5-very much).

The design of the final survey followed the data protection advice for social studies of the Norwegian Centre for Research Data (NSD). According to the $\mathrm{NSD}^{7}$, surveys that are distributed online do not need to be formally approved by an ethics committee if they comply with the following standards: (i) the survey does not include information that can identify individuals directly (e.g.name and surname, email) or indirectly (e.g. combination of questions such as postal code, age and marital status); and (ii) the survey will not be connected to identifying information about the respondent (e.g. IP address or email address). The final version of the survey complied with these requirements.

The survey was distributed using a snow-ball sampling technique: the link to the survey was distributed among authors' professional and personal contacts using email and social media (e.g. WhatsApp, Linkedln, Facebook, Instagram, Twitter), so recipients could re-forward within their contacts and social networks. The link to survey was also shared by authors' institutions social media. Once the survey was closed, answers were downloaded and deleted from the platform.

Data analysis. All the statistical analyses were performed in $\mathrm{R}$ (version 3.6.2.) using RStudio (66), considering a significant level of $p<0.05$. The values of the screening scales were transformed to categorical variables: the PHQ-2 and GAD-2 scales results (values from 0 to 6) were classified into two groups, applying a cut-off value of $\geq 3$, in accordance with studies that fixed scores 3-or-greater as a good cut-point for screening for potential cases of depression and anxiety $(62,63)$. The PHQ4 scale results (values from 0 to 12), which serves as marker of psychological distress, were transformed as None-to-minimal (values $\leq 2)$, Mild (3-5), Moderate (6-8) and Severe (9-12) (59).

To elucidate whether levels of lockdown (hypothesis 1), types of accessible outdoor spaces (hypothesis 2a) and types of views (hypothesis $2 \mathrm{~b}$ ) influenced mental health, Kruskal-Wallis Test followed by Dunn's Test for multiple comparisons was used for PHQ-4 (ordinal variable with >2 categories) and the Chi-squared test with pairwise comparison as post hoc test for PHQ-2 and GAD-2 (ordinal variable with 2 categories). The $p$-values of post hoc tests were adjusted with Bonferroni corrections. Mental health scale values were compared between levels of lockdown using the whole sample $(n=6,080)$, while for mental health scale values $v s$. types of accessible outdoor spaces and types of views, the subsample of respondents in Spain under Level 1 of lockdown $(n=3,403)$ was used. The level of lockdown in Spain was one of the most severe in Europe, and the high number of responses received from this country allowed for a more in-depth analysis, splitting responses according to types of views and types of accessible outdoors spaces. To determine the relative odds that individuals with meaningful symptoms of depression and anxiety (i.e. above the cut-off value of $\geq 3$ ), logistic GLMs were built.

To test hypothesis 1, models were built with PHQ-2 and GAD-2 results as a function of lockdown level, access to outdoor spaces and natural elements in the views from windows. Models were estimated for the whole sample and dividing the sample by severe lockdown (Level 1) and other types of lockdown (Level 2 and 3). The accessibility to outdoor spaces was introduced as a binary variable in the model, considering if respondents had access to any outdoor spaces or not, independently of the type. The information on views was transformed to a binary variable considering if the view included any natural element (no $=$ few views or urban views, yes $=$ rest of views).

To analyse if the effect of contact with nature on mental health varied depending on the type of views and type of accessible outdoor spaces (i.e. hypothesis 2), the subsample of respondents in Spain under Level 1 of lockdown was used. Logistic GLMs used PHQ-2 and GAD-

${ }^{7}$ https://nsd.no/personvernombud/en/help/research methods/online surveys.html 
2 results as dependent variables and two independent variables: 1) the type of accessible outdoor spaces, as a four-category variable (none, balcony, other private areas [e.g. private garden] and shared or public areas); and 2) the type of views that respondents could see from their lockdown residence, as a three-category variable considering the level of natural component (few views or urban views, mixed views and natural views).

All the models were later adjusted with 11 variables including sociodemographic characteristics, lockdown conditions and personal characteristics: age (18-25 years=reference), gender (female=reference), maximum education level achieved (primary or secondary=reference), whether employment situation changed after coronavirus outbreak (no=reference), income (transformed to categorical variable by estimating the ratio Income / per capita Gross Domestic Product [GDP], and with income/GDP $<2=$ reference), BRCS (numeric), whether respondents owned a pet that needed walking outside (no=reference), residence size (ratio of rooms per people in lockdown) and characteristics of the company during lockdown, such as whether respondent was alone (no=reference), with kids (no=reference), and with people with special care needs (no=reference). The variables were tested against multicollinearity estimating the Variance Inflation Factor (VIF) and assuming a threshold value of $<3(67)$. The logistic GLMs were built in R Studio (66) using the stats package (68). Odds ratios (ORs) and 95\% confidence intervals (Cls) were estimated with the questionr package (69), and the fit of the models as the Cox and Snell Pseudo $\mathrm{R}^{2}$ estimate using the DescTools package (70).

Finally, two additional analysis were performed to test hypothesis $2 \mathrm{a}$ and $2 \mathrm{~b}$ related with: a) respondent perceptions on the effect of views and outdoor spaces in coping with lockdown measures, depending on types of outdoor spaces and views; and b) the emotions that respondents mentioned as their most common feeling during lockdown and that better describe their character under normal circumstances. The self-reported effect of access to outdoor spaces was analysed with the responses to the Likert-scale question: To what extent do you think having physical access to these areas is helping you to better cope with the lockdown? The self-reported effect of views was analysed with the responses to: To what extent do you think having that view is helping you to better cope with the lockdown? To test for significant differences across types of accessible outdoor spaces and views, a one-way ANOVA was conducted, followed by post hoc Tukey's honestly significant difference (HSD) test. Two Ordinary Least Squares (OLS) regression models were built with the scores in the two Likert-scales as dependent variables, and 1) type of accessible outdoor area or 2) type of views as independent variable. Regressions were adjusted with the same 11 sociodemographic variables used in the above-mentioned GLMs. When analysing the perceived contribution of accessible outdoor spaces, individuals with no accessible outdoor spaces did not answer the question and therefore were excluded from the analyses.

Regarding changes in emotions, those mentioned by respondents under strict lockdowns as their most common feeling during lockdown were compared with the ones selected to describe their character under normal circumstances. Additional comparisons were performed to compare emotions of individuals with vs. without access to outdoor spaces and natural elements in views. Emotions were ordered according to the frequency they were mentioned using the tm package in $R(71,72)$, and later grouped according to the seven core emotions (SI Survey). To determine if frequency of core emotions changed after lockdown, and if differences exist depending on access to outdoor spaces and natural elements on views, the Chi-squared Test of Independence was used.

\section{Acknowledgments}

We acknowledge the essential contributions of the study participants in this research. SP was supported by a postdoctoral grant from the Department of Education of the Basque Government (Programme for the Specialization of Doctoral Researchers). The research was supported in part by: the European Union's Horizon 2020 research and innovation program under grant agreement No 774567 (H2020 SOPHIE Project) and No 666773 (H2020 BlueHealth Project); the UK Natural Environment Research Council (NERC) and the UK Research and Innovation's Global Challenges 
Research Fund (GCRF) for the Blue Communities Project. This is contribution ... from AZTI's Marine Research (Basque Research and Technology Alliance, BRTA).

\section{References}

1. L. Wang, Y. Wang, D. Ye, Q. Liu, Review of the 2019 novel coronavirus (SARS-CoV-2) based on current evidence. International Journal of Antimicrobial Agents, 105948 (2020).

2. S. G. Benzell, A. Collis, C. Nicolaides, Rationing social contact during the COVID-19 pandemic: Transmission risk and social benefits of US locations. Proc Natl Acad Sci USA, 202008025 (2020).

3. A. Tobías, Evaluation of the lockdowns for the SARS-CoV-2 epidemic in Italy and Spain after one month follow up. Science of The Total Environment 725, 138539 (2020).

4. S. Flaxman, et al., Estimating the effects of non-pharmaceutical interventions on COVID-19 in Europe. Nature (2020) https:/doi.org/10.1038/s41586-020-2405-7 (June 9, 2020).

5. C. Le Quéré, et al., Temporary reduction in daily global CO2 emissions during the COVID-19 forced confinement. Nat. Clim. Chang. (2020) https:/doi.org/10.1038/s41558-020-0797-x (June 9, 2020).

6. D. Helm, The Environmental Impacts of the Coronavirus. Environ Resource Econ 76, 21-38 (2020).

7. J. W. Goodell, COVID-19 and finance: Agendas for future research. Finance Research Letters, 101512 (2020).

8. E. A. Holmes, et al., Multidisciplinary research priorities for the COVID-19 pandemic: a call for action for mental health science. The Lancet Psychiatry 7, 547-560 (2020).

9. S. Pappa, et al., Prevalence of depression, anxiety, and insomnia among healthcare workers during the COVID-19 pandemic: A systematic review and meta-analysis. Brain, Behavior, and Immunity, S088915912030845X (2020).

10. N. Ozamiz-Etxebarria, M. Dosil-Santamaria, M. Picaza-Gorrochategui, N. Idoiaga-Mondragon, Stress, anxiety, and depression levels in the initial stage of the COVID-19 outbreak in a ppulation sample in the northern Spain. Cad. Saúde Pública 36, e00054020 (2020).

11. S. K. Brooks, et al., The psychological impact of quarantine and how to reduce it: rapid review of the evidence. The Lancet 395, 912-920 (2020).

12. N. Leigh-Hunt, et al., An overview of systematic reviews on the public health consequences of social isolation and loneliness. Public Health 152, 157-171 (2017).

13. K. J. Smith, C. Victor, Typologies of loneliness, living alone and social isolation, and their associations with physical and mental health. Ageing and Society 39, 1709-1730 (2019).

14. World Health Organization, "Depression and other common mental disorders: Global health estimates" (2017).

15. D. D. Fancourt, D. F. Bu, D. H. W. Mak, A. Steptoe, "Covid-19 social study" (Nuffield Foundation, 2020).

16. N. Balluerka Lasa, et al., "Las consecuencias psicológicas de la COVID-19 y el confinamiento" (Servicio Publicaciones de la Universidad del País Vasco, 2020).

17. I. Heinen, M. Bullinger, R.-D. Kocalevent, Perceived stress in first year medical students associations with personal resources and emotional distress. BMC Med Educ 17, 4 (2017).

18. R.-D. Kocalevent, M. Zenger, A. Hinz, B. Klapp, E. Brähler, Resilient coping in the general population: standardization of the brief resilient coping scale (BRCS). Health Qual Life Outcomes 15, 251 (2017).

19. B. Cartwright, M. White, T. Clitherow, Nearby Nature 'Buffers' the Effect of Low Social Connectedness on Adult Subjective Wellbeing over the Last 7 Days. IJERPH 15, 1238 (2018).

20. Y. Yang, et al., Viewing nature scenes reduces the pain of social ostracism. The Journal of Social Psychology, 1-19 (2020).

21. K. Samuelsson, S. Barthel, J. Colding, G. Macassa, M. Giusti, "Urban nature as a source of resilience during social distancing amidst the coronavirus pandemic" (Open Science Framework, 2020) https:/doi.org/10.31219/osf.io/3wx5a (July 10, 2020). 
22. M. Gascon, W. Zijlema, C. Vert, M. P. White, M. J. Nieuwenhuijsen, Outdoor blue spaces, human health and well-being: A systematic review of quantitative studies. International Journal of Hygiene and Environmental Health 220, 1207-1221 (2017).

23. G. N. Bratman, et al., Nature and mental health: An ecosystem service perspective. Sci. Adv. 5, eaax0903 (2019).

24. M. Gascon, et al., Mental Health Benefits of Long-Term Exposure to Residential Green and Blue Spaces: A Systematic Review. IJERPH 12, 4354-4379 (2015).

25. J. Thomsen, R. Powell, C. Monz, A Systematic Review of the Physical and Mental Health Benefits of Wildland Recreation. Journal of Park and Recreation Administration 36 (2018).

26. A. Borja, et al., Moving Toward an Agenda on Ocean Health and Human Health in Europe. Front. Mar. Sci. 7, 37 (2020).

27. R. Costanza, et al., Twenty years of ecosystem services: How far have we come and how far do we still need to go? Ecosystem Services 28, 1-16 (2017).

28. L. Keniger, K. Gaston, K. Irvine, R. Fuller, What are the Benefits of Interacting with Nature? IJERPH 10, 913-935 (2013).

29. $\mathrm{H}$. Kruize, et al., Exploring mechanisms underlying the relationship between the natural outdoor environment and health and well-being - Results from the PHENOTYPE project. Environment International 134, 105173 (2020).

30. M. P. White, S. Pahl, B. W. Wheeler, M. H. Depledge, L. E. Fleming, Natural environments and subjective wellbeing: Different types of exposure are associated with different aspects of wellbeing. Health \& Place 45, 77-84 (2017).

31. J. Roe, P. Aspinall, The restorative benefits of walking in urban and rural settings in adults with good and poor mental health. Health \& Place 17, 103-113 (2011).

32. M. G. Berman, et al., Interacting with nature improves cognition and affect for individuals with depression. Journal of Affective Disorders 140, 300-305 (2012).

33. M. P. White, et al., Spending at least 120 minutes a week in nature is associated with good health and wellbeing. Sci Rep 9, 7730 (2019).

34. D. Nutsford, A. L. Pearson, S. Kingham, F. Reitsma, Residential exposure to visible blue space (but not green space) associated with lower psychological distress in a capital city. Health \& Place 39, 70-78 (2016).

35. J. K. Garrett, et al., Urban blue space and health and wellbeing in Hong Kong: Results from a survey of older adults. Health \& Place 55, 100-110 (2019).

36. S. Dempsey, M. T. Devine, T. Gillespie, S. Lyons, A. Nolan, Coastal blue space and depression in older adults. Health \& Place 54, 110-117 (2018).

37. A. E. van den Berg, J. Maas, R. A. Verheij, P. P. Groenewegen, Green space as a buffer between stressful life events and health. Social Science \& Medicine 70, 1203-1210 (2010).

38. S. Xu, Y. Li, Beware of the second wave of COVID-19. The Lancet 395, 1321-1322 (2020).

39. K. Leung, J. T. Wu, D. Liu, G. M. Leung, First-wave COVID-19 transmissibility and severity in China outside Hubei after control measures, and second-wave scenario planning: a modelling impact assessment. The Lancet 395, 1382-1393 (2020).

40. S. de Bell, H. Graham, S. Jarvis, P. White, The importance of nature in mediating social and psychological benefits associated with visits to freshwater blue space. Landscape and Urban Planning 167, 118-127 (2017).

41. Z. S. Venter, D. N. Barton, V. Gundersen, H. Figari, Urban nature in a time of crisis: recreational use of green space increases during the COVID-19 outbreak in Oslo, Norway. 28 (2020).

42. E. Lange, P. V. Schaeffer, A comment on the market value of a room with a view. Landscape and Urban Planning 55, 113-120 (2001).

43. M. White, et al., Blue space: The importance of water for preference, affect, and restorativeness ratings of natural and built scenes. Journal of Environmental Psychology 30, 482-493 (2010).

44. G. MacKerron, S. Mourato, Happiness is greater in natural environments. Global Environmental Change 23, 992-1000 (2013).

45. A. Masten, A. Barnes, Resilience in Children: Developmental Perspectives. Children 5, 98 (2018). 
46. T. Kavčič, A. Avsec, G. Zager Kocjan, Psychological Functioning of Slovene Adults during the COVID-19 Pandemic: Does Resilience Matter? Psychiatr Q (2020) https:/doi.org/10.1007/s11126-020-09789-4 (July 20, 2020).

47. R. Lieb, E. Becker, C. Altamura, The epidemiology of generalized anxiety disorder in Europe. European Neuropsychopharmacology 15, 445-452 (2005).

48. C. Valiente, et al., "Síntomas de ansiedad, depresión y estrés postraumático ante el COVID19: prevalencia y predictores" (2020).

49. World Health Organization, "Mental health: A call for action by World Health Ministers" (2001).

50. M. Bartley, Unemployment and ill health: understanding the relationship. Journal of Epidemiology \& Community Health 48, 333-337 (1994).

51. R. Costanza, et al., The value of the world's ecosystem services and natural capital. Nature 387, 253-260 (1997).

52. Millennium Ecosystem Assessment, Ed., Ecosystems and human well-being: synthesis (Island Press, 2005).

53. R. Haines-Young, M. Potschin, Common International Classification of Ecosystem Services (CICES) V5.1 and guidance on the application of the revised structure. Fabis consulting (2018) (September 21, 2018).

54. A. Abraham, K. Sommerhalder, T. Abel, Landscape and well-being: a scoping study on the health-promoting impact of outdoor environments. International Journal of Public Health 55, 59-69 (2010).

55. J. Settele, S. Diaz, E. Brondizio, P. Daszak, COVID-19 Stimulus measures must save lives, protect livelihoods, and safeguard nature to reduce the risk of future pandemics. IPBES expert guest article (2020).

56. A. Hunt, P. Watkiss, Climate change impacts and adaptation in cities: a review of the literature. Climatic Change 104, 13-49 (2011).

57. B. Ma, T. Zhou, S. Lei, Y. Wen, T. T. Htun, Effects of urban green spaces on residents' wellbeing. Environment, Development and Sustainability 21, 2793-2809 (2019).

58. L. R. Elliott, et al., Research Note: Residential distance and recreational visits to coastal and inland blue spaces in eighteen countries. Landscape and Urban Planning 198, 103800 (2020).

59. K. Kroenke, R. L. Spitzer, J. B. W. Williams, B. Löwe, An Ultra-Brief Screening Scale for Anxiety and Depression: The PHQ-4. Psychosomatics 50, 613-621 (2009).

60. K. Kroenke, R. L. Spitzer, J. B. W. Williams, B. Löwe, The Patient Health Questionnaire Somatic, Anxiety, and Depressive Symptom Scales: a systematic review. General Hospital Psychiatry 32, 345-359 (2010).

61. J. García-Campayo, et al., The assessment of generalized anxiety disorder: psychometric validation of the Spanish version of the self-administered GAD-2 scale in daily medical practice. Health Qual Life Outcomes 10, 114 (2012).

62. K. Kroenke, R. L. Spitzer, J. B. W. Williams, P. O. Monahan, B. Löwe, Anxiety Disorders in Primary Care: Prevalence, Impairment, Comorbidity, and Detection. Ann Intern Med 146, 317 (2007).

63. K. Kroenke, R. L. Spitzer, Janet B. W. Williams, The Patient Health Questionnaire-2: Validity of a Two-Item Depression Screener. Medical Care 41, 1284-1292 (2003).

64. R. Plutchik, Emotions: a psychoevolutionary synthesis (Harper \& Row, 1980).

65. V. G. Sinclair, K. A. Wallston, The Development and Psychometric Evaluation of the Brief Resilient Coping Scale. Assessment 11, 94-101 (2004).

66. RStudio Team, RStudio: Integrated Development for R (RStudio, Inc., 2019).

67. A. F. Zuur, E. N. leno, C. S. Elphick, A protocol for data exploration to avoid common statistical problems: Data exploration. Methods in Ecology and Evolution 1, 3-14 (2010).

68. R Core Team, R: A language and environment for statistical computing ( $\mathrm{R}$ Foundation for Statistical Computing, 2019).

69. J. Barnier, F. Briatte, J. Larmarange, questionr: Functions to Make Surveys Processing Easier (2020).

70. A. Signorell, et mult. al., DescTools: Tools for Descriptive Statistics (2020).

71. I. Feinerer, K. Hornik, D. Meyer, Text Mining Infrastructure in R. J. Stat. Soft. 25 (2008). 
72. I. Feinerer, K. Hornik, tm: Text Mining Package (2019). 


\section{Figures and Tables}

Table 1. Distribution of responses by scores in the mental health scales and lockdown severity. Chi-squared test was used for PHQ-2 (Patient Health Questionnaire) and GAD-2 (Generalized Anxiety Disorder) values (ordinal variables with 2 levels), and Kruskal Wallis test for $P H Q-4$ values (ordinal variable with $>2$ levels). Different letters $(A, B)$ indicate significant differences $(p<0.05$ with Bonferroni correction) between groups, after the corresponding post hoc tests (pairwise comparison for PHQ-2 and GAD-2 and post hoc Dunn Test for PHQ-4).

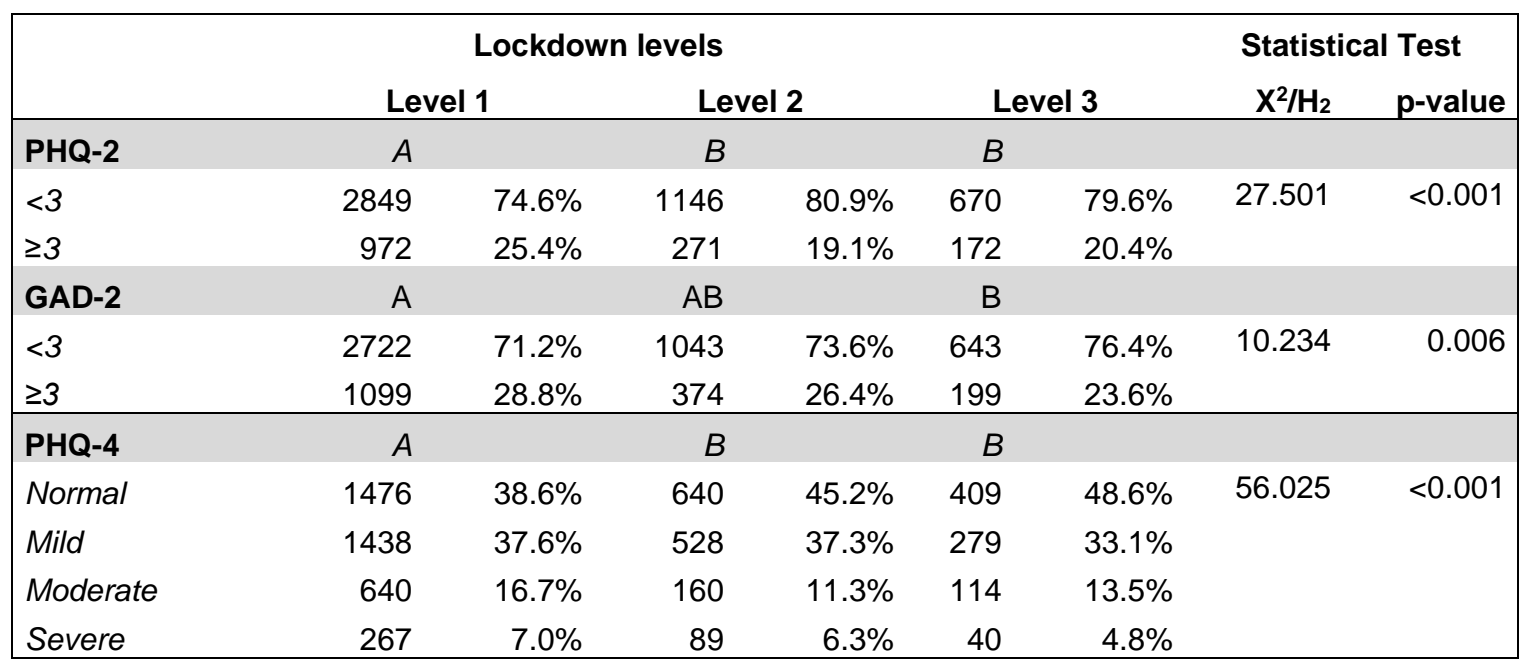


Table 2. Logistic Generalized Linear Models for depression and anxiety for the whole sample $(n=6,080)$, Level $1(n=3,821)$ and Level2\&3 $(n=2,259)$. OR $<1$ indicates a decrease in the likelihood of showing depression or anxiety symptoms; OR $>1$ equals to an increase in symptoms. $\mathrm{OR}=$ Odds ratio; $\mathrm{Cl}=$ Confident Interval; $\mathrm{GDP}=$ Gross Domestic Product; $\mathrm{PHQ}-2=$ Patient Health Questionnaire-2; GAD-2= Generalized Anxiety Disorder-2; ${ }^{*} \mathrm{p}<0.05 ;{ }^{* *} \mathrm{p}<0.01$; ${ }^{* *} \mathrm{p}<0.001$.

\begin{tabular}{|c|c|c|c|c|c|c|c|c|c|}
\hline & \multicolumn{3}{|c|}{ Whole sample } & \multicolumn{3}{|l|}{ Level 1} & \multicolumn{3}{|c|}{ Level 2\&3 } \\
\hline & OR & $\begin{array}{l}\text { 95\% Cl } \\
\text { Low }\end{array}$ & High & OR & \multicolumn{2}{|l|}{$95 \% \mathrm{Cl}$} & OR & $\begin{array}{l}95 \% \mathrm{Cl} \\
\text { Low }\end{array}$ & High \\
\hline \multicolumn{10}{|l|}{ PHQ-2 UNADJUSTED MODELS } \\
\hline $\begin{array}{l}\text { (Intercept) } \\
\text { Lockdown }\end{array}$ & $0.50^{\star * *}$ & 0.44 & 0.56 & $0.50^{\star * *}$ & 0.44 & 0.57 & $0.37^{\star \star \star}$ & 0.27 & 0.51 \\
\hline Level 2 & $0.78^{* *}$ & 0.67 & 0.91 & & & & & & \\
\hline $\begin{array}{l}\text { Level } 3 \\
\text { Outdoor views with natural elem. }\end{array}$ & $0.81^{*}$ & 0.68 & 0.98 & & & & & & \\
\hline $\begin{array}{l}\text { Yes } \\
\text { Access outdoors }\end{array}$ & $0.73^{* * *}$ & 0.65 & 0.83 & $0.74^{* * *}$ & 0.64 & 0.87 & $0.72^{\star *}$ & 0.58 & 0.89 \\
\hline Yes & $0.68^{\star * *}$ & 0.59 & 0.78 & $0.66^{\star * *}$ & 0.57 & 0.77 & 0.74 & 0.53 & 1.04 \\
\hline Pseudo $R^{2}$ & 0.015 & & & 0.013 & & & 0.006 & & \\
\hline \multicolumn{10}{|l|}{ PHQ-2 ADJUSTED MODELS } \\
\hline $\begin{array}{l}\text { (Intercept) } \\
\text { Lockdown }\end{array}$ & $24.31^{\star \star \star}$ & 15.45 & 38.40 & $31.50^{\star \star \star}$ & 18.20 & 54.93 & $9.67^{\star \star \star}$ & 4.03 & 23.27 \\
\hline Level 2 & $0.83^{*}$ & 0.70 & 0.98 & & & & & & \\
\hline $\begin{array}{l}\text { Level } 3 \\
\text { Outdoor views with natural elem. }\end{array}$ & 0.85 & 0.69 & 1.04 & & & & & & \\
\hline $\begin{array}{l}\text { Yes } \\
\text { Access outdoors }\end{array}$ & $0.77^{\star \star *}$ & 0.68 & 0.89 & $0.77^{* *}$ & 0.65 & 0.92 & $0.77^{*}$ & 0.61 & 0.97 \\
\hline $\begin{array}{l}\text { Yes } \\
\text { House Space (Rooms/person) }\end{array}$ & $0.72^{\star \star \star}$ & 0.61 & 0.83 & $0.67^{* \star *}$ & 0.57 & 0.80 & 0.91 & 0.63 & 1.31 \\
\hline $\begin{array}{l}\text { (numeric) } \\
\text { Gender }\end{array}$ & $0.91^{*}$ & 0.83 & 1.00 & 0.91 & 0.80 & 1.02 & 0.94 & 0.81 & 1.07 \\
\hline Male & $0.81^{* *}$ & 0.70 & 0.93 & $0.84^{*}$ & 0.70 & 0.99 & 0.78 & 0.61 & 1.00 \\
\hline $\begin{array}{l}\text { Other } \\
\text { Age }\end{array}$ & 0.64 & 0.30 & 1.26 & $0.38^{*}$ & 0.14 & 0.91 & 1.76 & 0.52 & 5.09 \\
\hline $26-35$ & $0.48^{* * *}$ & 0.39 & 0.59 & $0.44^{\star * *}$ & 0.34 & 0.57 & $0.56^{\star *}$ & 0.39 & 0.80 \\
\hline $36-45$ & $0.28^{* * *}$ & 0.22 & 0.35 & $0.25^{\star * *}$ & 0.19 & 0.33 & $0.34^{\star * *}$ & 0.23 & 0.51 \\
\hline $46-55$ & $0.20^{* * *}$ & 0.16 & 0.25 & $0.21^{* * *}$ & 0.16 & 0.27 & $0.18^{\star \star \star}$ & 0.11 & 0.28 \\
\hline $56-65$ & $0.19^{\star \star *}$ & 0.14 & 0.24 & $0.17^{* \star *}$ & 0.12 & 0.23 & $0.24^{\star \star \star}$ & 0.14 & 0.38 \\
\hline $\begin{array}{l}>65 \\
\text { Education level }\end{array}$ & $0.16^{\star \star \star}$ & 0.11 & 0.24 & $0.16^{* \star *}$ & 0.10 & 0.25 & $0.15^{\star \star \star}$ & 0.06 & 0.32 \\
\hline Proff. Educ. / University degree & 0.85 & 0.67 & 1.08 & 0.81 & 0.62 & 1.06 & 1.08 & 0.65 & 1.84 \\
\hline $\begin{array}{l}\text { Higher (Master, PhD) } \\
\text { Income (rank) }\end{array}$ & 0.83 & 0.64 & 1.07 & 0.81 & 0.60 & 1.09 & 0.97 & 0.59 & 1.65 \\
\hline income/GDP per capita $>2$ & 0.89 & 0.72 & 1.10 & 0.91 & 0.67 & 1.22 & 0.91 & 0.67 & 1.23 \\
\hline $\begin{array}{l}\text { no data } \\
\text { Change employment }\end{array}$ & 1.04 & 0.90 & 1.19 & 1.00 & 0.84 & 1.18 & 1.11 & 0.86 & 1.43 \\
\hline $\begin{array}{l}\text { Yes } \\
\text { Brief Resilience Coping Scale }\end{array}$ & $1.36^{\star *}$ & 1.09 & 1.69 & 1.27 & 0.98 & 1.65 & $1.62^{*}$ & 1.07 & 2.44 \\
\hline $\begin{array}{l}\text { (numeric) } \\
\text { Lockdown with kids }\end{array}$ & $0.83^{* * *}$ & 0.81 & 0.85 & $0.83^{* * *}$ & 0.80 & 0.85 & $0.84^{\star \star \star}$ & 0.81 & 0.88 \\
\hline $\begin{array}{l}\text { Yes } \\
\text { Lockdown alone }\end{array}$ & 0.89 & 0.76 & 1.05 & 0.89 & 0.73 & 1.09 & 0.91 & 0.68 & 1.22 \\
\hline $\begin{array}{l}\text { Yes } \\
\text { People with Special Care Needs }\end{array}$ & 1.20 & 0.96 & 1.51 & 1.14 & 0.83 & 1.54 & 1.35 & 0.95 & 1.90 \\
\hline $\begin{array}{l}\text { Yes } \\
\text { Pet needs walking }\end{array}$ & 1.18 & 0.95 & 1.45 & 0.99 & 0.76 & 1.29 & $1.67^{\star \star}$ & 1.15 & 2.40 \\
\hline Yes & $1.25^{\star *}$ & 1.06 & 1.48 & $1.34^{* \star}$ & 1.09 & 1.65 & 1.08 & 0.81 & 1.45 \\
\hline Pseudo $R^{2}$ & 0.119 & & & 0.131 & & & & & \\
\hline
\end{tabular}




\begin{tabular}{|c|c|c|c|c|c|c|c|c|c|}
\hline & \multicolumn{3}{|c|}{ Whole sample } & \multicolumn{3}{|l|}{ Level 1} & \multicolumn{3}{|c|}{ Level $2 \& 3$} \\
\hline & \multirow[t]{2}{*}{ OR } & $\begin{array}{r}95 \% \\
\text { Low } \\
\end{array}$ & \multirow[t]{2}{*}{ High } & OR & $\begin{array}{r}95^{\circ} \\
\text { Low }\end{array}$ & \% ${ }^{1} \mathrm{Cl}$ & OR & $\begin{array}{r}95 \% \\
\text { Low }\end{array}$ & High \\
\hline \multicolumn{8}{|l|}{ GAD-2 UNADJUSTED MODELS } & & \\
\hline (Intercept) & $0.54^{* \star \star}$ & 0.48 & 0.60 & $0.54^{* \star \star}$ & 0.47 & 0.61 & $0.48^{\star \star \star}$ & 0.35 & 0.64 \\
\hline Level 2 & 0.97 & 0.84 & 1.11 & & & & & & \\
\hline $\begin{array}{l}\text { Level } 3 \\
\text { Outdoor views with natural }\end{array}$ & $0.81^{*}$ & 0.68 & 0.97 & & & & & & \\
\hline $\begin{array}{l}\text { elem. } \\
\text { Yes } \\
\text { Access outdoors }\end{array}$ & $0.80^{\star \star \star}$ & 0.71 & 0.90 & $0.77^{\star \star *}$ & 0.66 & 0.89 & 0.88 & 0.73 & 1.07 \\
\hline Yes & $0.75^{\star \star \star}$ & 0.65 & 0.86 & $0.76^{\star \star \star}$ & 0.66 & 0.89 & $0.73^{*}$ & 0.53 & 1.00 \\
\hline Pseudo $R^{2}$ & 0.008 & & & 0.008 & & & 0.003 & & \\
\hline \multicolumn{10}{|l|}{ GAD-2 ADJUSTED MODELS } \\
\hline $\begin{array}{l}\text { (Intercept) } \\
\text { Lockdown }\end{array}$ & $7.45^{\star \star \star}$ & 4.90 & 11.35 & $7.54^{* \star \star}$ & 4.55 & 12.54 & $6.21^{* \star \star}$ & 2.77 & 13.94 \\
\hline Level 2 & 1.04 & 0.89 & 1.21 & & & & & & \\
\hline $\begin{array}{l}\text { Level } 3 \\
\text { Outdoor views with natural }\end{array}$ & 0.86 & 0.71 & 1.04 & & & & & & \\
\hline $\begin{array}{l}\text { Yes } \\
\text { Access outdoors }\end{array}$ & $0.83^{* *}$ & 0.73 & 0.94 & $0.78^{* *}$ & 0.67 & 0.91 & 0.91 & 0.74 & 1.12 \\
\hline $\begin{array}{l}\text { Yes } \\
\text { House Space (Rooms/person) }\end{array}$ & $0.75^{* * *}$ & 0.65 & 0.87 & $0.77^{\star *}$ & 0.66 & 0.90 & 0.72 & 0.52 & 1.01 \\
\hline $\begin{array}{l}\text { (numeric) } \\
\text { Gender }\end{array}$ & 1.01 & 0.93 & 1.10 & 0.98 & 0.87 & 1.09 & 1.08 & 0.96 & 1.23 \\
\hline Male & $0.55^{\star * \star}$ & 0.48 & 0.63 & $0.55^{\star \star \star}$ & 0.46 & 0.64 & $0.56^{\star \star \star}$ & 0.45 & 0.71 \\
\hline $\begin{array}{l}\text { Other } \\
\text { Age }\end{array}$ & 0.73 & 0.38 & 1.31 & 0.54 & 0.24 & 1.12 & 1.44 & 0.49 & 3.85 \\
\hline $26-35$ & $0.64^{\star \star \star}$ & 0.52 & 0.78 & $0.61^{* * *}$ & 0.48 & 0.79 & $0.66^{*}$ & 0.46 & 0.93 \\
\hline $36-45$ & $0.55^{\star}$ & 0.45 & 0.68 & $0.57^{\star \star \star}$ & 0.44 & 0.73 & $0.51^{\star *}$ & 0.35 & 0.75 \\
\hline $46-55$ & $0.41^{* * *}$ & 0.33 & 0.51 & $0.44^{\star \star *}$ & 0.34 & 0.57 & $0.34^{* * *}$ & 0.23 & 0.51 \\
\hline $56-65$ & $0.35^{\star * *}$ & 0.27 & 0.44 & $0.35^{\star * *}$ & 0.26 & 0.48 & $0.31^{* * *}$ & 0.20 & 0.49 \\
\hline $\begin{array}{l}>65 \\
\text { Education level }\end{array}$ & $0.28^{* * *}$ & 0.19 & 0.40 & $0.30^{* * *}$ & 0.20 & 0.46 & $0.22^{* * *}$ & 0.11 & 0.42 \\
\hline Proff. Educ. / University degree & 0.86 & 0.69 & 1.08 & 0.86 & 0.67 & 1.11 & 0.92 & 0.57 & 1.51 \\
\hline $\begin{array}{l}\text { Higher (Master, PhD) } \\
\text { Income (rank) }\end{array}$ & 0.81 & 0.64 & 1.04 & 0.79 & 0.59 & 1.04 & 0.98 & 0.62 & 1.60 \\
\hline income/GDP per capita >2 & 1.03 & 0.85 & 1.24 & 0.87 & 0.66 & 1.14 & 1.15 & 0.89 & 1.48 \\
\hline $\begin{array}{l}\text { no data } \\
\text { Change employment }\end{array}$ & 1.08 & 0.95 & 1.24 & 1.09 & 0.93 & 1.27 & 1.05 & 0.83 & 1.32 \\
\hline $\begin{array}{l}\text { Yes } \\
\text { Brief Resilience Coping Scale }\end{array}$ & 0.99 & 0.80 & 1.23 & 0.93 & 0.72 & 1.20 & 1.17 & 0.78 & 1.73 \\
\hline $\begin{array}{l}\text { (numeric) } \\
\text { Lockdown with kids }\end{array}$ & $0.88^{* \star *}$ & 0.86 & 0.90 & $0.89^{* * *}$ & 0.86 & 0.91 & $0.87^{\star \star \star}$ & 0.84 & 0.91 \\
\hline $\begin{array}{l}\text { Yes } \\
\text { Lockdown alone }\end{array}$ & 1.01 & 0.87 & 1.17 & 0.99 & 0.82 & 1.18 & 1.04 & 0.81 & 1.34 \\
\hline $\begin{array}{l}\text { Yes } \\
\text { People with Special Care } \\
\text { Needs }\end{array}$ & 0.82 & 0.66 & 1.02 & 0.76 & 0.56 & 1.03 & 0.88 & 0.63 & 1.23 \\
\hline $\begin{array}{l}\text { Yes } \\
\text { Pet needs walking }\end{array}$ & $1.31^{\star *}$ & 1.08 & 1.58 & 1.18 & 0.93 & 1.49 & $1.64^{\star *}$ & 1.18 & 2.28 \\
\hline Yes & $1.24^{\star *}$ & 1.07 & 1.45 & $1.27^{*}$ & 1.05 & 1.55 & 1.19 & 0.92 & 1.52 \\
\hline Pseudo $\mathbf{R}^{2}$ & 0.074 & & & 0.080 & & & 0.067 & & \\
\hline
\end{tabular}


Table 3. Comparison of PHQ-4 (Patient Health Questionnaire), PHQ-2 and GAD-2 (Generalized Anxiety Disorder) values between types of views and types of accessible outdoor spaces. The subsample of people in Spain and in Level 1 (3393 obs.) was used. The statistical test performed were Chi-squared test for GAD-2 and PHQ-2 values and Kruskal Wallis test for PHQ-4 values. Different letters $(A, B)$ indicate significant differences $(p<0.05)$ between groups (i.e. lockdown levels), after pairwise comparison for PHQ-2 and GAD-2 and after post hoc Dunn Test for PHQ-

4. The post hoc tests' p-values were calculated with Bonferroni correction for multiple-comparisons.

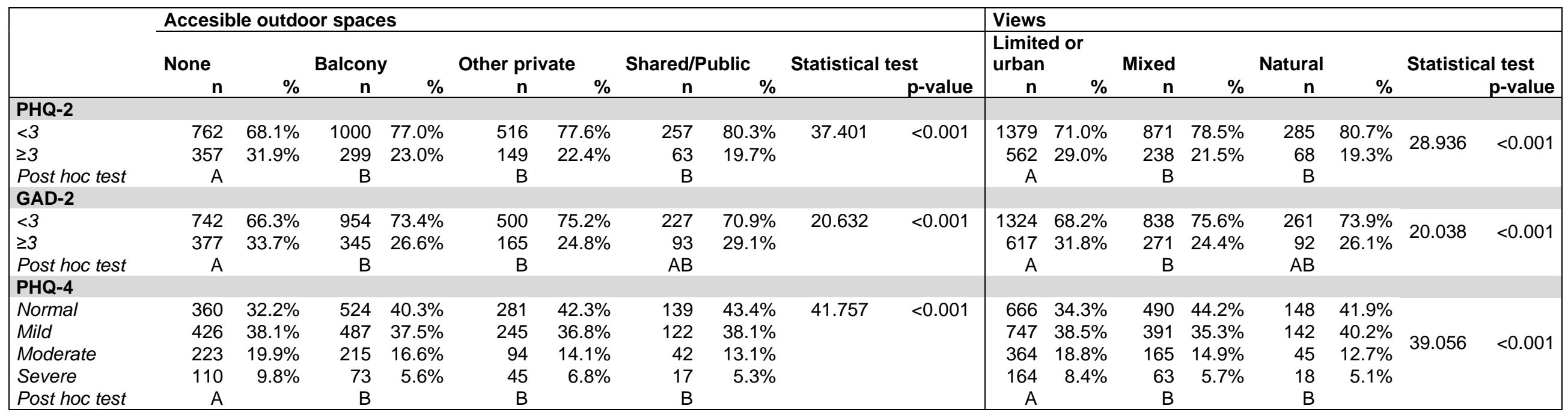


Table 4: Self-reported contribution of views and accessible outdoor spaces to cope with lockdown situation. Using the subsample of people in Spain and in Level 1 (3,393 obs.), selfreported contribution was captured through Likert-scale scores (from 0 "not at all" to 5 "very much"). Different lettering $(A, B, C)$ indicates significant differences for the Tukey's post hoc test.

\begin{tabular}{|lrrrrrr|}
\hline & & & & $\begin{array}{c}\text { ANOVA } \\
\text { (n) }\end{array}$ & $\begin{array}{c}\text { Tukey's } \\
\text { post } \\
\text { hoc }\end{array}$ \\
\hline Accessible outdoor spaces & & Mean & SD & F & p-value \\
Only balcony & 1299 & 4.15 & 1.02 & & & $\mathrm{~A}$ \\
Other private spaces & 665 & 4.50 & 0.94 & 25.96 & $<0.001$ & $\mathrm{~B}$ \\
Shared and/or public spaces & 320 & 4.27 & 1.14 & & & $\mathrm{~A}$ \\
None & 1119 & 4.08 & 1.05 & & & \\
\hline Views & & & & & & \\
Limited or urban & 1941 & 2.98 & 1.28 & & & $\mathrm{~A}$ \\
Mixed & 1109 & 4.09 & 1.01 & 533.5 & $<0.001$ & $\mathrm{~B}$ \\
Natural & 353 & 4.67 & 0.67 & & & $\mathrm{C}$ \\
\hline
\end{tabular}

${ }^{8}$ Data of people with no accessible outdoor spaces was not considered in the ANOVA, as they did not answer the question on the self-reported contribution of outdoor spaces. 
Figure 1. Percentages of the core-emotions mentioned by the people in Spain under lockdown Level 1, to describe their mood before and during lockdown. A total of five comparisons were done: i) Before lockdown vs. during lockdown; ii) People with limited outdoor views vs. people with outdoors views before lockdown and iii) during lockdown; iv) People with no accessible outdoor spaces vs. people with accessible outdoor spaces before lockdown and v) during lockdown. Significant differences after the Chi-squared test results are presented as ${ }^{* * *} p<0.001,{ }^{* *} p<0.01$, ${ }^{*} \mathrm{p}<0.05$.

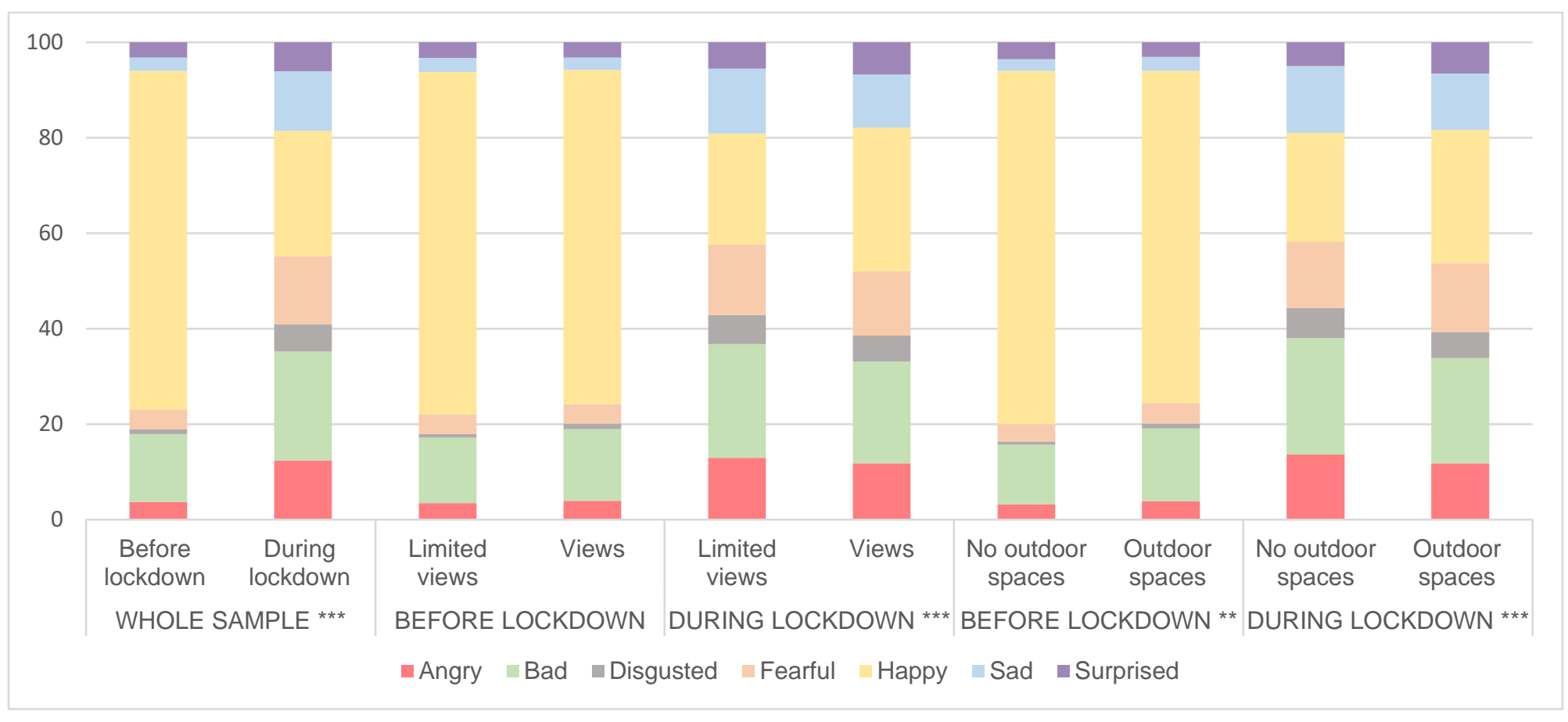

